\title{
REDUCTION OF SCHMIDT PHOTOGRAPHS WITH THE AID OF REFERENCE MARKS ON THE CURVED PLATE
}

P. LACROUTE

Strasbourg Observatory, France

\begin{abstract}
A method is proposed for improving the astrometric accuracy obtainable from curved-field Schmidt plates. A reseau of small crosses in a $10 \mathrm{~mm}$-spaced rectangular grid is imposed on the plate while still curved in its holder. The reseau is calibrated by photography of a field of reference stars with known positions. The method eliminates the troublesome errors arising from uncertainty in the nature of deformation of the curved plates.
\end{abstract}

\section{DISCUSSION}

Dieckvoss: The whole field of the Bergedorf Schmidt cannot be used. I recommend a device such as Prof. Lacroute's for studying the irregular bending effects.

Lacroute: I, too, would like to try to improve it by using marks. 